\title{
STANDARD IDEALS IN WEIGHTED ALGEBRAS OF KORENBLYUM AND WIENER TYPES
}

\author{
JOSÉ E. GALÉ and ANTONI WAWRZYŃCZYK*
}

\begin{abstract}
We introduce two types of Banach algebras of analytic functions on the unit disc which can be seen as weighted versions of closed primary ideals of the Korenblyum and (analytic) Wiener algebras, respectively. Such types of algebras arise in connection with convolution Banach algebras of Sobolev type, on the positive half-line, and their discrete analogues defined in terms of higher order differences. We show that all closed ideals are standard for algebras in the first class, and that closed ideals with countable hull are standard in algebras of the second class.
\end{abstract}

\section{Introduction}

Let $\mathscr{B}$ be a (non necessarily unital) Banach algebra of bounded holomorphic functions on the unit disc $D:=\{z \in C:|z|<1\}$, endowed with the pointwise operations and the norm $\|\cdot\|_{\mathscr{B}}$. The problem of describing the closed ideals in $\mathscr{B}$ is generally not a simple question, but it is possible to give a complete classification of ideals in some important examples. In any case, a basic notion to consider in this setting is that one of standard (closed) ideal, see [2]. Here we widen slightly the concept of standard ideal as follows.

Fix a nonnegative integer $k$. Let $\left(X_{n}\right)_{n=0}^{k}$ be a family of closed subsets of T with $X_{0} \subseteq X_{1} \subseteq \cdots \subseteq X_{k}$, where T denotes, as usually, the unit circle. Assume that for every $f \in \mathscr{B}$ and every $n=0,1, \ldots, k$ the derivative $f^{(n)}$ extends continuously to $\mathrm{T} \backslash X_{n}$ (so that the spectrum of regular maximal ideals of $\mathscr{B}$ is assumed to be $\overline{\mathrm{D}} \backslash X_{0}$, in particular). For a closed ideal $I$ in $\mathscr{B}$ and $0 \leq n \leq k$, put

$$
Z^{n}(I):=\left\{z \in \overline{\mathrm{D}} \backslash X_{n}: f(z)=f^{\prime}(z)=\cdots=f^{(n)}(z)=0, \forall f \in I\right\},
$$

and

$$
E_{n}(I):=Z^{n}(I) \cap \mathrm{T}
$$

(the set $Z(I):=Z^{0}(I)$ is called the hull of $I$ ). Let $Q_{I}$ be the greatest inner common divisor of all nonzero functions in $I$ (see [12, p. 85]). Then we call

\footnotetext{
* The research of the first named author has been supported by the Project MTM2007-61446, DGI-FEDER, of the MCYTS, Spain, and Project E-64, D.G. Aragón, Spain.

Received 22 September 2009, in final form 20 July 2010.
} 
the ideal I standard in $\mathscr{B}$ if

$$
I=\left\{f \in \mathscr{B}: Q_{I} \text { divides } f \text { and } f^{(n)}=0 \text { on } E_{n}(I),(0 \leq n \leq k)\right\} .
$$

There are important classes of Banach algebras as the previous $\mathscr{B}$, with $X_{k}=\emptyset$, where all closed ideals are standard; see the introduction and references of [2]. Among them, there is the Korenblyum algebra $A^{k}(\mathrm{D})$, formed by definition by all holomorphic functions in $\mathrm{D}$ such that $f^{(n)}$ extends continuously to $\mathrm{T}$ for every $n=0,1, \ldots, k$.

There are on the other hand interesting Banach algebras $\mathscr{B}$, also with $X_{k}=$ $\emptyset$, which contain non-standard ideals and where, for instance, closed ideals with countable hull are standard [2]. Among these, we pay attention on the Banach algebra $A^{+}(\mathrm{T})$, which we call here the analytic Wiener algebra, formed by all functions $f$ in $A^{0}$ (D) with absolutely convergent Taylor series $f(z)=$ $\sum_{m=0}^{\infty} a_{m} z^{m},(|z| \leq 1)$. The norm in $A^{+}(\mathrm{T})$ is

$$
\|f\|_{A^{+}(\mathrm{T})}:=\sum_{m=0}^{\infty}\left|a_{m}\right|, \quad f \in A^{+}(\mathrm{T}) .
$$

In the present paper we deal with weighted versions of both Korenblyum and analytic Wiener algebras, separately. More precisely, for a nonnegative integer $k$, let $\mathfrak{U}^{(k)}(\mathrm{D})$ denote the Banach algebra of all functions $f$ in the disc algebra $A(\mathrm{D}):=A^{0}(\mathrm{D})$ such that $f(1)=0$,

$$
\left(1-z^{2}\right)^{n} f^{(n)} \in A(\mathrm{D}) \quad \text { and } \quad \lim _{z \rightarrow \pm 1}\left(1-z^{2}\right)^{n} f^{(n)}(z)=0 \quad(1 \leq n \leq k) .
$$

The norm in this algebra is given by

$$
\|f\|:=\sum_{n=0}^{k}\left\|\left(1-z^{2}\right)^{n} f^{(n)}\right\|_{\infty}, \quad f \in \mathfrak{U}^{(k)}(\mathrm{D}) .
$$

We can see that the algebra $\mathfrak{U}^{(k)}(\mathrm{D})$ is a Korenblyum type algebra with weight $\left(1-z^{2}\right)^{n}$ in the $n$-th derivative, $1 \leq n \leq k$.

Similarly, we define $\mathscr{H}_{1}^{(k),+}(\mathrm{T})$ as the subalgebra of $A(\mathrm{D})$ formed by all functions $f$ in $A(D)$ such that

$$
(1-z)^{n} f^{(n)}(z) \in A^{+}(\mathrm{T}) \quad(n=0,1, \ldots, k),
$$

provided with the Banach algebra norm

$$
\|f\|_{1,(k)}:=\sum_{n=0}^{k}\left\|(1-z)^{n} f^{(n)}\right\|_{A^{+}(\mathrm{T})} .
$$


In this case the algebra $\mathscr{H}_{1}^{(k),+}(\mathrm{T})$ is a Wiener type algebra with weights $(1-z)^{n}$ in the corresponding derivatives.

The algebras $\mathfrak{H}^{(k)}(\mathrm{D})$ and $\mathscr{H}_{1}^{(k),+}(\mathrm{T})$ arise in relation with convolution Banach algebras of Sobolev type on the positive half-line and their discrete analogues, respectively. This is as follows.

Let $\mathscr{T}_{+}^{(k)}\left(t^{k}\right)$ denote the Banach space obtained as the completion of the space $C_{c}^{(\infty)}[0, \infty)$ of test functions on $[0, \infty)$, with respect to the norm

$$
\|f\|:=\int_{0}^{\infty}\left|f^{(k)}(t)\right| t^{k} d t, \quad f \in C_{c}^{(\infty)}[0, \infty) .
$$

The space $\mathscr{T}_{+}^{(k)}\left(t^{k}\right)$ is a non-unital commutative semisimple Banach algebra for the convolution on $(0, \infty)$, and a subalgebra of $L^{1}\left(\mathrm{R}^{+}\right) \equiv \mathscr{T}_{+}^{(0)}\left(t^{0}\right)$. It was introduced in [1], in the realm of integrated/distribution operator semigroup theory, and its applications to ill-posed Cauchy problems. From the point of view of the Banach algebra theory, a further study has been carried out in [6], [7], [8], even in a fractional derivation setting. Among other properties, we have that the character space of $\mathscr{T}_{+}^{(k)}\left(t^{k}\right)$ can be identified with $\overline{\mathrm{C}^{+}}$and its Gelfand transform coincides with the Laplace transform $\mathscr{L}$. In fact the image of $\mathscr{T}_{+}^{(k)}\left(t^{k}\right)$ by $\mathscr{L}$ lies in the Banach algebra $\mathfrak{H}^{(k)}\left(\mathrm{C}^{+}\right)$of analytic functions $F$ on $\mathrm{C}^{+}:=\{\lambda \in \mathrm{C}: \Re \lambda>0\}$ such that $\lambda^{j} F^{(j)}(\lambda)$ extends continuously up to the boundary $i \mathrm{R}$ of $\mathrm{C}^{+}$for $j=0,1, \ldots, k$, and satisfies

$$
\lim _{\lambda \rightarrow 0} \lambda^{j} F^{(j)}(\lambda)=0, \quad(1 \leq j \leq k) ; \quad \lim _{\lambda \rightarrow \infty} \lambda^{j} F^{(j)}(\lambda)=0, \quad(0 \leq j \leq k) .
$$

The norm in $\mathfrak{U}^{(k)}\left(\mathrm{C}^{+}\right)$is given by

$$
\|F\|:=\sum_{j=0}^{n} \max _{\Re i \geq 0}|\lambda|^{j}\left|F^{(j)}(\lambda)\right|, \quad F \in \mathfrak{U}^{(k)}\left(\mathrm{C}^{+}\right) .
$$

Of course the restricted Laplace transform

$$
\mathscr{L}: \mathscr{T}_{+}^{(k)}\left(t^{k}\right) \rightarrow \mathfrak{U}^{(k)}\left(\mathrm{C}^{+}\right)
$$

is a bounded Banach algebra homomorphism. Moreover, $\mathscr{L}$ has dense range: $\overline{\mathscr{T}_{+}^{(k)}\left(t^{k}\right)}=\mathfrak{U}^{(k)}\left(\mathrm{C}^{+}\right)$; see [8].

As regards the ideal theory in $\mathscr{T}_{+}^{(k)}\left(t^{k}\right)$, a Nyman-type characterization of dense ideals has been given in [7] which raises the question of whether or not $I=\mathscr{L}^{-1}(\overline{\mathscr{L}(I)})$, for every closed ideal $I$ in $\mathscr{T}_{+}^{(k)}\left(t^{k}\right)$ with empty zero-set $Z(\mathscr{L}(I))$, where the closure is taken in $\mathfrak{U}^{(k)}\left(\mathrm{C}^{+}\right)$. Such an equality holds in the case when $k=0$, even for more general closed ideals (see [2], [5], [15]). 
In order to eventually undertake the investigation of closed ideals in $\mathscr{T}_{+}^{(k)}\left(t^{k}\right)$ (a task which is not simple even for $k=0$, see the above references and [3], [10]) it seems appropriate to understand first the closed ideals of $\mathfrak{A}^{(k)}\left(\mathrm{C}^{+}\right)$. This question looks interesting in its own.

The Möbius mapping $\mu: z \rightarrow(1-z)^{-1}(1+z)$ transforms the disc $\mathrm{D}$ onto the half-plane $C^{+}$and $\bar{D}$ onto $\overline{C^{+}} \cup\{\infty\}$. By composition of $\mu$ with the functions of $\mathfrak{U}^{(k)}\left(\mathrm{C}^{+}\right)$we get exactly the Banach algebra $\mathfrak{U}^{(k)}(\mathrm{D})$ introduced formerly. In this way, we are led to study the closed ideals of $\mathfrak{Y}^{(k)}(D)$.

Analogously, the algebra $\mathscr{H}_{1}^{(k),+}(\mathrm{T})$ appears in association with a discrete analogue of the convolution algebra $\mathscr{T}_{+}^{(k)}\left(t^{k}\right)$ which we introduce here using difference operators of higher degree: For a complex sequence $a$ and integers $n, m \geq 0$ put

$$
\Delta^{0} a(n):=a(n) ; \quad \Delta a(n):=a(n)-a(n+1) ; \quad \Delta^{m+1}:=\Delta^{1} \circ \Delta^{m} .
$$

Let $\tau^{k}\left(n^{k}\right)$ be the space of sequences $a$ such that

$$
\lim _{n \rightarrow \infty} a(n)=0 \quad \text { and } \quad \sum_{n=1}^{\infty}\left|\Delta^{k} a(n)\right| n^{k}<\infty .
$$

The space $\tau^{k}\left(n^{k}\right)$ is a Banach algebra with respect to the convolution product of sequences, subalgebra of $\tau^{0}\left(n^{0}\right) \equiv \ell^{1}\left(\mathrm{~N}_{0}\right)$, and the norm

$$
\|a\|:=|a(0)|+\sum_{n=1}^{\infty}\left|\Delta^{k} a(n)\right| n^{k}, \quad a \in \tau^{k}\left(n^{k}\right) .
$$

The character space of $\tau^{k}\left(n^{k}\right)$ is (topologically) isomorphic to the closed unit $\overline{\mathrm{D}}$, and the Gelfand transform of each $a \in \tau^{k}\left(n^{k}\right)$ coincides with the FourierTaylor series of $a$. Moreover, the Banach algebras $\tau^{k}\left(n^{k}\right)$ and $\mathscr{H}_{1}^{(k),+}(\mathrm{T})$ are isomorphic via that Taylor series mapping. The above facts are proven in Sections 4 and 5 below.

The algebra $\tau^{k}\left(n^{k}\right)$ seems to be of interest in its own. There are spaces of sequences of higher (fractional) bounded variation, defined in terms of higher fractional difference operators, which have been considered in the context of Fourier multiplier theory, see [9] (although our space $\tau^{k}\left(n^{k}\right)$ admits a direct extension, for any positive real number $k$, defined in terms of fractional difference powers, we are not going further in this direction here). In this way the convolution structure is incorporated to that framework. In a forthcoming paper we will find out the possible links between closed ideals of $\tau^{k}\left(n^{k}\right)$ and those of $\mathscr{T}_{+}^{(k)}\left(t^{k}\right)$ (for $k=0$, see [11]). 
It turns out that we can get information about closed ideals in the algebras $\mathscr{B}=\mathfrak{U}^{(k)}(\mathrm{D}), \mathscr{H}_{1}^{(k),+}(\mathrm{T})$ by application of the same method in both cases. It consists of taking a suitable multiplication operator $M: \mathscr{B} \rightarrow M(\mathscr{B})$ such that $M(\mathscr{B})$ is an easily identifiable closed ideal of a convenient Banach algebra $\mathscr{A}$ on the unit disc, and $\mathscr{B}$ becomes Banach $\mathscr{A}$-module isomorphic to $M(\mathscr{B})$.

For $\mathscr{B}=\mathfrak{A l}^{(k)}(\mathrm{D})$ we take $M$ as the multiplication by $(1+z)^{k}(1-z)^{k}$, so that $\mathscr{A}$ is the Korenblyum algebra. Then we can derive that all closed ideals of $\mathfrak{H}^{(k)}(\mathrm{D})$ are standard (Theorem 3.2) from the fact that every closed ideal in $\mathscr{A}=A^{k}(\mathrm{D})$ is standard [13]. See Section 1 below.

For $\mathscr{B}=\mathscr{H}_{1}^{(k),+}(\mathrm{T})$ the multiplication operator is realized by $M \equiv(1-z)^{k}$. Then the algebra $\mathscr{A}$ is a weighted version, $\mathscr{A}=A_{k}^{+}(\mathrm{T})$, of the analytic Wiener algebra, whence we obtain that any closed ideal in $\mathscr{H}_{1}^{(k),+}(\mathrm{T})$ having countable hull is standard, this time because all such ideals in $A_{k}^{+}(\mathrm{T})$ are standard, see [2] and Section 1 below.

The organization of the paper is as follows. Firstly, Section 1 is about preliminaries, where we expose some material concerning Korenblyum and analytic Wiener algebras, and their ideals. Then Section 2 is devoted to preparatory results on a certain auxiliary Banach algebra, denoted by $\mathscr{H}_{w}^{(k)}(\mathrm{D})$, which will be subsequently applied in Section 3 to establish the main result on the closed ideals of the Banach algebras $\mathfrak{H}^{(k)}(\mathrm{D})$ and $\mathfrak{A l}^{(k)}\left(\mathrm{C}^{+}\right)$, Theorem 3.2 and Corollary 3.3. In Section 4, we introduce the Banach space $\tau^{k}\left(n^{k}\right)$, give its basic properties, and prove that it is a Banach algebra for the convolution, Theorem 4.5. Then Section 5 contains the characterization of the range of $\tau^{k}\left(n^{k}\right)$ as $\mathscr{H}_{1}^{(k),+}(\mathrm{T})$, through the Fourier-Taylor series map (Theorem 5.4). The proof relies upon the invertibility of a certain matrix, Lemma 5.2. Finally, in Section 6 we apply the aforementioned general method, involving a (multiplication operator) module isomorphism, to show that the closed ideals with countable hull in $\tau^{k}\left(n^{k}\right) \simeq \mathscr{H}_{1}^{(k),+}(\mathrm{T})$ are standard, Theorem 6.1 and Theorem 6.2.

At this point, an attentive reader will have already noticed that by a Banach algebra we mean here any Banach space which, in addition, is endowed with a jointly continuous multiplication (so the submultiplicative norm constant need not be 1). Throughout the paper we will make use of the constant convention: the same letter, say $C$, is used for possibly different constants even in the same argument.

\section{Preliminaries: Standard ideals in the Korenblyum and Wiener algebras}

Let $H^{\infty}(\mathrm{D})$ be the Hardy space of bounded analytic functions on D. As is well known, for every function $f \in H^{\infty}(\mathrm{D})$ there exists the limit $f\left(e^{i \theta}\right):=$ $\lim _{r \rightarrow 1^{-}} f\left(r e^{i \theta}\right)$ for almost all $\theta \in[-\pi, \pi) \equiv \mathrm{T}:=\{z \in \mathrm{C}:|z|=1\}$. 
Recall that a function $\psi$ in $H^{\infty}(\mathrm{D})$ is inner if $\|\psi\|_{\infty} \leq 1$ and $\left|\psi\left(e^{i \theta}\right)\right|=1$ a. e. on $[-\pi, \pi)$. For an arbitrary family $\mathfrak{\mho}$ of inner functions there exists a unique inner function $Q$ which divides $f$ in the sense that $f / Q$ belongs to $H^{\infty}$ (D) for every $f \in \mathfrak{F}$, and such that every inner function which divides each element of $\mathfrak{F}$ also divides $Q$. The function $Q$ is called the greatest inner common divisor (g.c.d., for short) of the family $\mathfrak{\mho}$; see [12, p. 85] An analytic function $\phi \in$ is called outer if it satisfies

$$
\phi(z)=c \exp \left(\frac{1}{2 \pi} \int_{0}^{2 \pi} \frac{e^{i \theta}+z}{e^{i \theta}-z} \log \phi\left(e^{i \theta}\right) d \theta\right), \quad z \in \mathrm{D},
$$

where $c$ is a constant of absolute value 1 and the function $\phi$ is measurable on T with $\log \phi$ integrable. Every element $g$ of $H^{\infty}$ factorizes into $g=g^{i} g^{o}$, where $g^{i}$ is inner and $g^{o}$ is outer.

We next recall how the above notions go into the ideal theory of the Korenblyum algebra. Let $A(\mathrm{D})$ be the disc algebra, formed by the analytic functions on $\mathrm{D}$ which admit continuous extension to all of $\overline{\mathrm{D}}$, endowed with the supnorm $\|\cdot\|_{\infty}$. More generally, we denote by $A^{k}(\mathrm{D})$ the Korenblyum algebra of functions $f$ on $\mathrm{D}$ such that $f^{(n)} \in A(\mathrm{D})$ for every $0 \leq n \leq k$. In this case the algebra norm is given by

$$
\|f\|=\sum_{n=0}^{k}\left\|f^{(n)}\right\|_{\infty} .
$$

For $g \in A^{k}(\mathrm{D}), n=0,1, \ldots, k$, and an arbitrary ideal $I$ in $A^{k}(\mathrm{D})$ we define

$$
E_{k}(g)=\left\{z \in \mathrm{T} \mid g(z)=g^{\prime}(z)=\cdots=g^{(k)}(z)=0\right\}
$$

and

$$
E_{n}(I)=\bigcap_{g \in I} E_{n}(g)
$$

Obviously, $E_{k}(I) \subseteq E_{k-1}(I) \subseteq \cdots \subseteq E_{0}(I)$. Let us choose now an inner function $Q$ and an arbitrary collection of closed sets

$$
\mathscr{E}: E_{k} \subseteq E_{k-1} \subseteq E_{1} \subseteq \cdots \subseteq E_{0} \subseteq \mathrm{T} .
$$

Define

$$
J(Q ; \mathscr{E})=\left\{g \in A^{k}(\mathrm{D}): Q \text { divides } g^{i}, E_{n} \subset E_{n}(g), 0 \leq n \leq k\right\} .
$$

Then $J(Q ; \mathscr{C})$ is a standard closed ideal in $A^{k}(\mathrm{D})$, possibly trivial. The fact that the closed ideals of $A^{k}(\mathrm{D})$ are all standard was proved by Korenblyum in his classical paper [13]. 
THEOREM 1.1 ([13]). For every closed ideal $I \subseteq A^{k}(\mathrm{D})$ there exists an inner function $Q_{I}$ such that

$$
I=J\left(Q_{I} ; E_{0}(I), E_{1}(I) \ldots E_{k}(I)\right) .
$$

The above theorem tells us that every closed ideal in $A^{k}(\mathrm{D})$ is completely determined by the collection of the common zeros of its elements and derivatives and by the inner function $Q_{I}$ obtained as the g. c. d. of $I$. As a corollary of the theorem we obtain that an ideal $I$ in $A^{k}(\mathrm{D})$ is dense if and only if the set of common zeros is empty and $Q_{I}=1$. Hence, a function $f$ generates a dense principal ideal in $A^{k}(\mathrm{D})$ if and only if $f$ is outer without zeros.

The standard ideals are defined in the same way as above for the analytic Wiener algebra $A^{+}(\mathrm{T})$, which, we recall, is formed by the functions in $A(\mathrm{D})$ whose Fourier-Taylor coefficient series are in $\ell^{1}$. More generally, we denote by $A_{k}^{+}(\mathrm{T})$ the subalgebra of those functions $f$ in $A^{+}(\mathrm{T})$ such that

$$
\sum_{n=0}^{\infty}|\hat{f}(n)|\left(1+n^{k}\right)<\infty .
$$

With pointwise operations and the norm defined by the above series, $A_{k}^{+}(\mathrm{T})$ is a Banach algebra. As a matter of fact, $f \in A_{k}^{+}(\mathrm{T})$ if and only if $f^{(j)} \in A^{+}(\mathrm{T})$ for every $j=0,1, \ldots, k$, and the norm previously indicated is equivalent to $\sum_{j=0}^{\infty}\left\|f^{(j)}\right\|_{A^{+}(\mathrm{T})}$.

Not every closed ideal in $A_{k}^{+}(\mathrm{T})$ is standard, see [3] for $k=0$, but we have the following partial result in the positive direction.

Theorem 1.2 ([2, Theorem 3.1]). If I is a closed ideal of $A_{k}^{+}(\mathrm{T})$ with countable hull then $I$ is standard, that is, there exists an inner function $Q_{I}$ such that

$$
I=J\left(Q_{I} ; E_{0}(I), \ldots, E_{k}(I)\right) .
$$

\section{Banach algebras module-isomorphic to primary ideals in the Korenblyum algebra}

Fix $w \in \mathrm{T}$. The following simple lemma gives us the key tool for the section. It may be well known, but we include a sketch of proof for the sake of completeness.

Lemma 2.1. Let $\varphi \in H^{\infty}(\mathrm{D})$ be such that $\varphi^{(j)}$ extends continuously to $V \cap \mathrm{T}$ for each $j=0,1, \ldots, k$, where $V=\overline{\mathrm{D}} \cap B$ for some disc $B$ centered at $w$. Let $n \leq k$ and suppose that $\varphi(w)=\varphi^{\prime}(w)=\cdots=\varphi^{(n-1)}(w)=0$. Then

$$
\lim _{z \rightarrow w} \frac{\varphi^{(j)}(z)}{(w-z)^{n-j}}=\frac{(-1)^{n-j}}{(n-j) !} \varphi^{(n)}(w) .
$$

for all $j=0,1, \ldots, n-1$. 
Proof. Since for every $j=0,1, \ldots, n-1$ we have that $\varphi^{(n)}=\left(\varphi^{(j)}\right)^{(n-j)}$, it is clearly enough to show the formula for $j=0$.

Take $m$ such that $1 \leq m \leq n$. Since $\varphi^{(m)}$ is continuous and $\varphi^{(m-1)}(w)=0$ we have, integrating by parts,

$$
\varphi(z)=\int_{[w, z]} \frac{(z-\lambda)^{n-1}}{(n-1) !} \varphi^{(n)}(\lambda) d \lambda \quad(z \in V \backslash\{w\})
$$

Hence

$$
\begin{aligned}
& \left|\frac{\varphi(z)}{(w-z)^{n}}-\frac{(-1)^{n}}{n !} \varphi^{(n)}(w)\right| \\
& \quad \leq \frac{1}{|w-z|^{n}}\left|\int_{[w, z]}(z-\lambda)^{n-1}\left[\varphi^{(n)}(\lambda)-\varphi^{(n)}(w)\right] d \lambda\right| \\
& \quad \leq \max _{\lambda \in[w, z]}\left|\varphi^{(n)}(\lambda)-\varphi^{(n)}(w)\right| \rightarrow 0, \quad \text { as } \quad z \rightarrow w
\end{aligned}
$$

as was required.

Fix a natural number $k$. We define $\mathscr{H}_{w}^{(k)}(\mathrm{D})$ as the space of holomorphic functions $f$ on the unit disc $\mathrm{D}$ such that $(w-z)^{n} f^{(n)} \in A$ (D) for $0 \leq n \leq k$, and

$$
\lim _{w \rightarrow z}(w-z)^{n} f^{(n)}(z)=0 \quad \text { for } \quad 1 \leq n \leq k
$$

Equipped with the norm $\|f\|=\sum_{n=0}^{k}\left\|(w-z)^{n} f^{(n)}\right\|_{A(\mathrm{D})}$, the Leibniz rule applies to show that the space $\mathscr{H}_{w}^{(k)}$ (D) is a unital commutative Banach algebra which contains continuously $A^{k}(\mathrm{D})$ as a subalgebra. In fact, $\mathscr{H}_{w}^{(k)}(\mathrm{D})$ is a Banach $A^{k}(\mathrm{D})$-module.

Let us also define the space $\mathscr{I}_{w}^{(k-1)}(\mathrm{D})$ by

$$
\mathscr{I}_{w}^{(k-1)}(\mathrm{D}):=\left\{g \in A^{k}(\mathrm{D}) \mid g^{(j)}(w)=0(j=0,1 \ldots, k-1)\right\} .
$$

Clearly, $\mathscr{I}_{w}^{(k-1)}(\mathrm{D})$ is the standard closed ideal $J(1 ;\{w\}, \ldots,\{w\}, \emptyset)$ of the Korenblyum algebra $A^{k}(\mathrm{D})$, so it is in particular a Banach $A^{k}(\mathrm{D})$-module.

Proposition 2.2. The multiplication operator

$$
\Psi: f \mapsto(w-z)^{k} f, \mathscr{H}_{w}^{(k)}(\mathrm{D}) \rightarrow A(\mathrm{D})
$$

defines a Banach module isomorphism between the Banach $A^{k}(\mathrm{D})$-modules $\mathscr{H}_{w}^{(k)}(\mathrm{D})$ and $\mathscr{I}_{w}^{(k-1)}(\mathrm{D})$. 
Proof. Let $f$ be holomorphic in D such that $\left(1-z^{n}\right) f^{(n)} \in A(\mathrm{D})$ for $0 \leq n \leq k$ (in particular this is satisfied by functions in $\mathscr{H}_{w}^{(k)}(\mathrm{D})$ ). By the Leibniz formula

(3) $\left((w-z)^{k} f\right)^{(n)}(z)=\sum_{j=0}^{n}(-1)^{j}\left(\begin{array}{l}n \\ j\end{array}\right)\left(\begin{array}{l}k \\ j\end{array}\right)(w-z)^{k-n}(w-z)^{n-j} f^{(n-j)}(z)$,

where each term is clearly null at $z=w$ if $n<k$, and if $j<k$ when $n=k$. It follows that there exists the $\operatorname{limit}_{\lim } \rightarrow w\left((w-z)^{k} f\right)^{(k)}(z)=f(w)$ and so $\varphi:=(w-z)^{k} f$ belongs to $\mathscr{I}_{w}^{(k-1)}(\mathrm{D})$. The continuity of $\Psi$ also follows by the above formula.

We are going to show that $\Psi$ is surjective. Thus take $\varphi \in \mathscr{I}_{w}^{(k-1)}(\mathrm{D})$ and define $f(z):=\varphi(z)(w-z)^{-k}$ for $z \in \overline{\mathrm{D}} \backslash\{w\}$. Obviously, each derivative $f^{(n)}$ extends continuously to $\overline{\mathrm{D}} \backslash\{w\}$ for every $n=0,1, \ldots, k$. Moreover, it follows directly from Lemma 2.1 applied to the case $n=k$ that the function $f$ extends continuously to $z=w$ as well. To show the extension of the derivatives of $f$ we introduce the auxiliary function

$$
\phi(z)=\varphi(z)+\frac{(-1)^{k-1}}{k !} \varphi^{(k)}(w)(w-z)^{k}, \quad z \in \mathrm{D} .
$$

It satisfies that $\phi(w)=\phi^{\prime}(w)=\cdots=\phi^{(k)}(w)=0$, while $f-(w-z)^{-k} \phi$ is a constant function. Hence, for $1 \leq n \leq k$, we obtain

$$
\begin{aligned}
(w-z)^{n} f^{(n)}(z) & =(w-z)^{n}\left(\phi(z)(w-z)^{-k}\right)^{(n)}(z) \\
& =\sum_{j=0}^{n}\left(\begin{array}{l}
n \\
j
\end{array}\right) \frac{k !}{(n-j) !} \phi^{(j)}(z)(w-z)^{-(k-j),}
\end{aligned}
$$

so that $\lim _{z \rightarrow w}(w-z)^{n} f^{(n)}(z)=0$ by Lemma 2.1; that is, $f \in \mathscr{H}_{w}^{(k)}(\mathrm{D})$ and we have that the operator $\Psi$ is bijective. Since $\Psi$ is also continuous it is a Banach space isomorphism.

Proposition 2.2 contains implicitly the following result.

COROLlaRY 2.3. Let $f$ be holomorphic in $\mathrm{D}$ such that $(w-z)^{n} f^{(n)} \in A(\mathrm{D})$ for $n=0,1, \ldots, k$. Then

$$
\lim _{z \rightarrow w}(w-z)^{n} f^{(n)}(z)=0 \quad \text { for } n=1, \ldots, k .
$$

Proof. As at the beginning of the proof of Proposition 2.2, the function $\varphi:=(w-z)^{k} f$ belongs to $\mathscr{I}_{w}^{(k-1)}(\mathrm{D})$. Now, by the same proposition, $f=$ $\Psi^{-1}(\varphi) \in \mathscr{H}_{w}^{(k)}(\mathrm{D})$. 
REMARK 2.4. The above corollary is somehow interesting. It says that, in the definition of the Banach algebra $\mathscr{H}_{w}^{(k)}(\mathrm{D})$, the only necessary assumption is $(w-z)^{n} f^{(n)} \in A(\mathrm{D})$ for $0 \leq n \leq k$, since this implies automatically that $(w-z)^{n} f^{(n)}$ vanishes at $w$ for $n=1, \ldots, k$. Such an implication is clear when $f$ is analytic at $w$ because of the local factorization principle.

Proposition 2.2 also implies that there is a bijective correspondence between the closed ideals of the algebra $\mathscr{H}_{w}^{(k)}(\mathrm{D})$ and the closed ideals in $A^{k}(\mathrm{D})$ contained in the ideal $\mathscr{I}_{w}^{(k-1)}(\mathrm{D})$. In particular the standard ideals contained in $\mathscr{I}_{w}^{(k-1)}(\mathrm{D})$ are of the form $I=J\left(Q, E_{0}, \ldots, E_{k-1}, E_{k}\right)$, where $w \in E_{k-1}$. Thus we have the following.

THEOREM 2.5. If I is a closed ideal in $\mathscr{H}_{w}^{(k)}(\mathrm{D})$ then $(w-z)^{k} I$ is a closed ideal in $A^{k}(\mathrm{D})$. Conversely, if $J \subset \mathscr{I}_{w}^{(k-1)}(\mathrm{D})$ is a closed ideal in $A^{k}(\mathrm{D})$ then the closed ideal I generated in $\mathscr{H}_{w}^{(k)}(\mathrm{D})$ by $J$ satisfies $(w-z)^{k} I=J$.

In particular all closed ideals in $\mathscr{H}_{w}^{(k)}(\mathrm{D})$ are standard, that is, they are of the form $J\left(Q ; \mathfrak{S}_{0}, \ldots, \mathfrak{S}_{k}\right)$ where $\mathfrak{S}_{0}$ is a closed subset of $\mathrm{T}, \mathfrak{S}_{n}$ is a relatively closed subset of $\mathrm{T} \backslash\{w\}$ for $1 \leq n \leq k$, and $\mathfrak{G}_{k} \subseteq \cdots \subseteq \mathfrak{E}_{0}$.

Proof. By Proposition 2.2 we know that for every closed ideal $I \subseteq \mathscr{H}_{w}^{(k)}(\mathrm{D})$ the set $(w-z)^{k} I$ belongs to $\mathscr{I}_{w}^{(k-1)}(\mathrm{D})$ and is a closed ideal of $A^{k}(\mathrm{D})$. Therefore it remains to prove that every closed subideal of $\mathscr{I}_{w}^{(k-1)}(\mathrm{D})$ can be represented in this form. If $J$ is such an ideal then it is of the form $J=J\left(Q ; E_{0}, E_{1}, \ldots, E_{k}\right)$ where $w \in E_{k-1}$. Denote by $I$ the closed ideal generated in the algebra $\mathscr{H}_{w}^{(k)}(\mathrm{D})$ by $J$. All elements of $I$ have the inner factor divisible by $Q$, hence this function is the greatest inner common divisor for elements of $I$ as well. The elements of $\mathscr{H}_{w}^{(k)}(\mathrm{D})$ are holomorphic functions of class $C^{(k)}$ on $\mathrm{T} \backslash\{w\}$. For every $n=0,1, \ldots, k$ and $z_{0} \in \mathrm{T} \backslash\{w\}$ the condition $\varphi^{(j)}\left(z_{0}\right)=0$ $(0 \leq j \leq n)$ is satisfied for all $\varphi=\psi \in J$ if and only if it is satisfied for all $\varphi=f \in I$. The set $\tilde{J}=(w-z)^{k} I$ is a closed ideal of $A^{k}(\mathrm{D})$ with the same data $Q, E_{0}, E_{1}, \ldots, E_{k}$ as $J$, so $\tilde{J}=J$. Moreover, the above argument tells us that $I=J\left(Q ; \mathfrak{S}_{0}, \ldots, \mathfrak{S}_{k}\right)$ if we take $\breve{S}_{0}=E_{0}$ and $\mathfrak{E}_{n}=E_{n} \backslash\{w\}$ $(1 \leq n \leq k)$.

Theorem 2.5 allows us to determine also the dense ideals of the algebra $\mathscr{H}_{w}^{(k)}(\mathrm{D})$.

Corollary 2.6. An ideal I is dense in $\mathscr{H}_{w}^{(k)}(\mathrm{D})$ if and only if the elements of I have no common zero in $\overline{\mathrm{D}}$ and there is no non-trivial common inner divisor of the elements of $I$.

Proof. An ideal $I$ is dense in $\mathscr{H}_{w}^{(k)}(\mathrm{D})$ if and only if $(w-z)^{k} I$ is dense in $\mathscr{I}_{w}^{(k)}(\mathrm{D})$. This holds if and only if $(w-z)^{k} I$ has no non-trivial common inner 
divisor and the unique common zero of elements of $(w-z)^{k} I$ is the point $w$. Then the elements of $I$ have no common zero.

Denote by $\mathscr{A}_{w}^{(k)}$ (D) the maximal ideal of $\mathscr{H}_{w}^{(k)}(\mathrm{D})$ consisting of functions which vanish at $w$. Note that every ideal of the algebra $\mathscr{A}_{w}^{(k)}(\mathrm{D})$ is at the same time an ideal of the algebra $\mathscr{H}_{w}^{(k)}(\mathrm{D})$. As another application of Lemma 2.1 and Theorem 2.5 we obtain a characterization of the closed ideals of $\mathscr{A}_{w}^{(k)}(\mathrm{D})$. Details of the proof are left to the reader.

Corollary 2.7. If $J=J\left(Q, E_{0}, \ldots, E_{k}\right)$ is a standard ideal in $A^{k}(\mathrm{D})$ such that $w \in E_{k}$ then the closed ideal generated in $\mathscr{A}_{w}^{(k)}(\mathrm{D})$ by $J$ is equal to $(w-z)^{-k} J$.

Conversely, all closed ideals I in $\mathscr{A}_{w}^{(k)}(\mathrm{D})$ are of this form; in particular $I=J\left(Q ; \mathfrak{S}_{0}, \ldots, \mathfrak{S}_{k}\right)$ where $\mathfrak{S}_{n}$ is a relatively closed subset of $\mathrm{T} \backslash\{w\}$ for $1 \leq n \leq k$, $\mathfrak{S}_{0}$ is a closed subset of $\mathrm{T}$ with $w \in \mathfrak{G}_{0}$, and $\mathfrak{S}_{k} \subseteq \cdots \subseteq \mathfrak{S}_{0}$.

Theorem 2.5 and Corollary 2.7 will be used together in the next section to get the characterization of closed ideals in $\mathfrak{U}^{(n)}(\mathrm{D})$.

\section{Closed ideals in Korenblyum-like algebras}

We show in this section that the closed ideals of the Banach algebra $\mathfrak{H}^{(k)}(\mathrm{D})$ are standard. Recall the definition of $\mathfrak{U}^{(k)}(\mathrm{D})$ :

For a nonnegative integer $k$, the Banach algebra $\mathfrak{U}^{(k)}(\mathrm{D})$ is formed by those functions $f$ in the disc algebra $A(D)$ such that $f(1)=0$,

$$
\left(1-z^{2}\right)^{n} f^{(n)} \in A(\mathrm{D}) \quad \text { and } \quad \lim _{z \rightarrow \pm 1}\left(1-z^{2}\right)^{n} f^{(n)}(z)=0 \quad(1 \leq n \leq k) \text {, }
$$

and is endowed with the norm given by

$$
\|f\|:=\sum_{n=0}^{k}\left\|\left(1-z^{2}\right)^{n} f^{(n)}\right\|_{\infty}, \quad f \in \mathfrak{U}^{(k)}(\mathrm{D}),
$$

and the pointwise operations. Notice that the elements of $\mathfrak{A l}^{(k)}(\mathrm{D})$ behave as functions of $\mathscr{H}_{-1}^{(k)}(\mathrm{D})$ in a neighbourhood of -1 and as functions of $\mathscr{A}_{1}^{(k)}(\mathrm{D})$ close to the point 1 . Moreover, $\mathscr{A}_{1}^{(k)}(\mathrm{D})$ is a subalgebra of $\mathfrak{U}^{(k)}(\mathrm{D})$.

Every ideal $I$ in $\mathfrak{H}^{(k)}(\mathrm{D})$ is invariant under the multiplication by elements of $\mathscr{H}_{-1}^{(k)}(\mathrm{D})$ since for each $f \in \mathscr{H}_{-1}^{(k)}(\mathrm{D})$ the function $f-f(1)$ belongs to $\mathfrak{U}^{(k)}(\mathrm{D})$ and, for arbitrary $g \in I$,

$$
f g=(f-f(1)) g+f(1) g \in I .
$$

In particular the closed ideals of $\mathfrak{H}^{(k)}(\mathrm{D})$ are Banach $A^{k}(\mathrm{D})$-modules and Banach $\mathscr{A}_{1}^{(k)}(\mathrm{D})$-modules. 
Next, we characterize the closed ideals of $\mathfrak{U}^{(k)}(\mathrm{D})$. The proof relies upon the existence of a Banach $A^{k}(\mathrm{D})$-module isomorphism of the algebra with a certain ideal of $A^{k}(\mathrm{D})$, and the Korenblyum's description of closed ideals of $A^{k}(\mathrm{D})$. The isomorphism is the content of the following result.

ThEOREM 3.1. For $\mathfrak{Y}^{(k)}(\mathrm{D})$ as above we have:

(a) The multiplication operator

$$
\Phi: f \mapsto(1+z)^{k}(1-z)^{k} f, \mathfrak{H}^{(k)}(\mathrm{D}) \rightarrow A^{k}(\mathrm{D})
$$

is a Banach $A^{k}(\mathrm{D})$-module isomorphism from $\mathfrak{H}^{(k)}(\mathrm{D})$ onto the standard closed ideal $\mathscr{J}^{(k)}(\mathrm{D}):=J(1 ;\{-1,1\}, \ldots,\{-1,1\},\{1\})$ of $A^{k}(\mathrm{D})$.

(b) The map $\Phi$ determines a one-to-one correspondence between closed ideals of $\mathfrak{U}^{(k)}(\mathrm{D})$ and the ideals of $A^{k}(\mathrm{D})$ of the form $J\left(Q ; E_{0}, \ldots, E_{k-1}\right.$, $\left.E_{k}\right) \subseteq A^{k}(\mathrm{D})$ such that $-1 \in E_{k-1}$ and $1 \in E_{k}$.

Proof. The Leibniz rule for derivatives implies that the space $(1-z)^{k} \mathfrak{Q}^{(k)}(\mathrm{D})$ is contained in $\mathscr{H}_{-1}^{(k)}(\mathrm{D})$. By applying Lemma 2.1 we then have that

$$
(1-z)^{k} \mathfrak{Q}^{(k)}(\mathrm{D})=\left\{\varphi \in \mathscr{H}_{-1}^{(k)}(\mathrm{D}) \mid \varphi(1)=\varphi^{\prime}(1)=\cdots=\varphi^{(k)}(1)=0\right\} .
$$

The latter space is the ideal $J(1 ;\{1\}, \ldots,\{1\})$ in $\mathscr{H}_{-1}^{(k)}(\mathrm{D})$ and multiplication by $(1+z)^{k}$ maps this ideal onto the standard ideal $\mathscr{J}^{(k)}(\mathrm{D})$ of $A^{k}(\mathrm{D})$, according to Theorem 2.5. Since $\Phi$ is continuous and bijective from $\mathfrak{U}^{(k)}(\mathrm{D})$ onto the closed ideal $\mathscr{J}^{(k)}(\mathrm{D})$, it is a Banach isomorphism by the open mapping theorem. Then $\Phi$ transforms closed ideals $I \subseteq \mathfrak{A}^{(k)}(\mathrm{D})$ onto closed ideals of $A^{k}(\mathrm{D})$ contained in the standard ideal $\mathscr{J}^{(k)}(\mathrm{D})$.

Conversely, if $J \subseteq \mathscr{J}^{(k)}$ (D) is a closed ideal in $A^{k}(\mathrm{D})$ then by Theorem 2.5 $J$ generates in $\mathscr{H}_{-1}^{(k)}(\mathrm{D})$ an ideal $J_{1}$ whose elements satisfy $f(1)=f^{\prime}(1)=$ $\cdots=f^{(k)}(1)=0$ and such that $(1+z)^{k} J_{1}=J$. By Lemma 2.1, taking $I=(1-z)^{-k} J_{1}$ we obtain an ideal in $\mathfrak{U}^{(k)}(\mathrm{D})$ that satisfies $\Phi(I)=J$.

To obtain a more explicit description of the closed ideals in $\mathfrak{H}^{(k)}(\mathrm{D})$ we refer to the definiton of zero-sets of order $k$ as it was given in the Introduction. So, for $g \in \mathfrak{U}^{(k)}(\mathrm{D})$ and an arbitrary closed ideal $I$ of $\mathfrak{U}^{(k)}(\mathrm{D})$, put

$$
\begin{aligned}
& \mathfrak{S}_{n}(g):=\left\{z \in \mathrm{T} \backslash\{-1,1\}: g^{(j)}(z)=0(0 \leq j \leq n)\right\} \quad \text { if } 1 \leq n \leq k, \\
& \mathfrak{S}_{0}(g):=\{z \in \mathrm{T} \backslash\{1\}: g(z)=0\}, \quad \text { and } \\
& \mathfrak{S}_{n}(I):=\bigcap_{g \in I} \mathfrak{S}_{n}(g) .
\end{aligned}
$$


THEOREM 3.2. For every closed ideal $I \subseteq \mathfrak{Q}^{(k)}(\mathrm{D})$ there exist an inner function $Q_{I}$ and a descending collection $\mathfrak{S}_{0} \supseteq \mathfrak{S}_{1} \supseteq \ldots \supseteq \mathfrak{S}_{k}$ of subsets of $\mathrm{T}$, where $\mathfrak{S}_{0}$ is a relatively closed subset of $\mathrm{T} \backslash\{1\}$ and $\mathfrak{S}_{n}$, for $1 \leq n \leq k$, is a relatively closed subset of $\mathrm{T} \backslash\{-1,1\}$, such that

$$
I=\left\{g \in \mathfrak{U}^{(k)}(\mathrm{D}) \mid Q_{I} \text { divides } g^{i} \text { and } \mathfrak{S}_{n} \subseteq \mathfrak{S}_{n}(g), 0 \leq n \leq k\right\} .
$$

Proof. Let $I$ be a closed ideal in $\mathfrak{H}^{(k)}(\mathrm{D})$. By Theorem 3.1,

$$
J:=(1+z)^{k}(1-z)^{k} I=J\left(Q ; E_{0}(J), E_{1}(J), \ldots, E_{k}(J)\right),
$$

for some inner function $Q$, where $1 \in E_{k}(J),-1 \in E_{k-1}(J)$. Put $E_{n}=E_{n}(J)$ and $\mathfrak{G}_{n}:=E_{n} \backslash\{-1,1\}$ for $0 \leq n \leq k$.

Note that $(1+z)^{k}(1-z)^{k}$ is an outer function. Thus for any function $g$ in $\mathfrak{H}^{(k)}(\mathrm{D})$ we have that

$$
\begin{aligned}
g \in I & \Leftrightarrow \varphi:=(1+z)^{k}(1-z)^{k} g \in J \\
& \Leftrightarrow Q \text { divides } \varphi^{i} \text { and } E_{n}(\varphi) \supseteq E_{n}(0 \leq n \leq k) \\
& \Leftrightarrow Q \text { divides } g^{i} \text { and } \breve{\complement}_{n}(g) \supseteq \breve{S}_{n}(0 \leq n \leq k),
\end{aligned}
$$

as we wanted to show.

The above result tells us that $\mathfrak{H}^{(k)}(\mathrm{D})$ is another example of a (non-unital) Banach algebra on the unit disc with all closed ideals of standard form, see [2].

Next we translate the previous results to the setting of the right-hand halfplane, which was one of our initial motivation to write this paper.

The Möbius map $\tau: \lambda \rightarrow(\lambda-1)(\lambda+1)^{-1}$ transforms $C^{+}$onto the disc $\mathrm{D}$ and $\overline{C^{+}} \cup\{\infty\}$ onto $\bar{D}$. The superposition with $\tau$ converts function algebras on the disc into function algebras on the half-plane. For a given space of functions $\mathscr{F}(\mathrm{D})$ we denote by $\mathscr{F}\left(\mathrm{C}^{+}\right)$the space of functions $f \circ \tau, f \in \mathscr{F}(\mathrm{D})$. In particular, let define $\mathfrak{U}^{(k)}\left(\mathrm{C}^{+}\right):=\mathfrak{H}^{(k)}(\mathrm{D}) \circ \tau$ and $\mathscr{J}^{(k)}\left(\mathrm{C}^{+}\right):=\mathscr{J}^{(k)}(\mathrm{D}) \circ \tau$. Note that, via composition with $\tau$, the functions $1+z$ and $1-z$ on the disc correspond to the functions $2 \lambda(\lambda+1)^{-1}$ and $2(\lambda+1)^{-1}$ on $\mathrm{C}^{+}$respectively. Then, it is straightforward to give an internal description of the functions which belong to $\mathfrak{U}^{(k)}\left(\mathrm{C}^{+}\right)$: The Banach algebra $\mathfrak{I}^{(k)}\left(\mathrm{C}^{+}\right)$consists of all holomorphic functions $F: \mathrm{C}^{+} \rightarrow \mathrm{C}$ such that $\lambda^{n} F^{(n)}$ extends continuously to $i \mathrm{R}$ for $0 \leq n \leq$ $k$, and

$$
\lim _{\lambda \rightarrow 0} \lambda^{n} F^{(n)}(\lambda)=0 \quad(1 \leq n \leq k) \quad \text { and } \quad \lim _{\lambda \rightarrow \infty} \lambda^{n} F^{(n)}(\lambda)=0 \quad(0 \leq n \leq k) .
$$


The norm in $\mathfrak{A C}^{(k)}\left(\mathrm{C}^{+}\right)$is given by $\|F\|=\sum_{n=0}^{k} \sup _{\lambda \in \mathrm{C}^{+}}|\lambda|^{n}\left|F^{(n)}(\lambda)\right|,(F \in$ $\left.\mathfrak{U}^{(k)}\left(\mathrm{C}^{+}\right)\right)$, and, as a straightforward calculation shows, the mapping $f \mapsto$ $F=f \circ \tau, \mathfrak{H}^{(k)}(\mathrm{D}) \rightarrow \mathfrak{U}^{(k)}\left(\mathrm{C}^{+}\right)$is a Banach algebra isomorphism.

Recall that a holomorphic function $F$ on $\mathrm{C}^{+}$is called inner (resp. outer) if $F \circ \tau$ is inner (resp. outer) in $\mathrm{D}$, see [12]. The subsets $\mathscr{E}_{n}(F) \subseteq i \mathrm{R}$, for $F \in \mathfrak{A}^{(k)}\left(\mathrm{C}^{+}\right)$, in the following statement are defined in a similar manner to the subsets $\mathfrak{S}_{n}(g) \subseteq \mathrm{T}$, for $g \in \mathfrak{U}^{(k)}(\mathrm{D})$.

COROLlaRY 3.3. For every ideal I $\subseteq \mathfrak{U}^{(k)}\left(\mathrm{C}^{+}\right)$there exists an inner function $G_{I}$ and a descending collection $\mathscr{E}_{0} \supseteq \mathscr{E}_{1} \supseteq \ldots \supseteq \mathscr{E}_{k}$ of subsets of $i \mathrm{R}$, where $\mathscr{E}_{0}$ is closed in $i \mathrm{R}$ and $\mathscr{E}_{n}$, for $1 \leq n \leq k$, is a relatively closed subset of $i \mathrm{R} \backslash\{0\}$, such that

$$
\begin{array}{r}
I=\left\{F \in \mathfrak{U}^{(k)}\left(\mathrm{C}^{+}\right) \mid G_{I}\right. \text { divides the inner } \\
\text { factor of } \left.F, \mathscr{E}_{n} \subseteq \mathscr{E}_{n}(F), 0 \leq n \leq k\right\} .
\end{array}
$$

From here we can give descriptions of the dense ideals in $\mathfrak{U}^{(k)}\left(\mathrm{C}^{+}\right)$.

Corollary 3.4. An ideal $I \subset \mathfrak{H}^{(k)}\left(\mathrm{C}^{+}\right)$is dense if and only if the elements of I have no common zero in $\overline{\mathrm{C}^{+}}$and the only common inner divisor of the elements of $I$ is the function 1. A function $F \in \mathfrak{\mathfrak { U } ^ { ( k ) }}\left(\mathrm{C}^{+}\right)$generates the algebra if and only if $F$ is outer and never vanishes.

The preceding corollary can alternatively be derived from [7, Theorem 3.1].

Corollary 3.5. An ideal I $\subset \mathfrak{H}^{(k)}\left(\mathrm{C}^{+}\right)$is dense if and only if the elements of I have no common zero in $\overline{\mathrm{C}^{+}}$and for every $a>0$ the space $e^{a z}$ I contains unbounded functions.

Proof. Suppose that $I$ is a dense ideal in $\mathfrak{A}^{(k)}\left(\mathrm{C}^{+}\right)$. Obviously $\mathscr{Z}(I)=\emptyset$, where $\mathscr{Z}(I):=\left\{\lambda \in \overline{\mathrm{C}^{+}}: f(\lambda)=0\right.$ for all $\left.f \in I\right\}$. Suppose that for some $a>0$ the space $e^{a z} I$ is formed exclusively by bounded functions. Every element $F$ of $I$ is of the form $F=e^{-a z} f$ where $f$ is analytic and bounded in $\mathrm{C}^{+}$.

Now, notice that, if $F_{n}=e^{-a z} f_{n} \rightarrow G$ in $\mathfrak{A}^{(k)}\left(\mathrm{C}^{+}\right)$then the sequence $\left(F_{n}\right)$ converges uniformly on $\overline{\mathrm{C}}^{+}$in particular, so it is a Cauchy sequence uniformly on $\overline{\mathrm{C}}^{+}$. Thus we have

$$
0=\lim _{m, n \rightarrow \infty} \sup _{x \in \mathrm{R}}\left|e^{-i a x} f_{n}(i x)-e^{-i a x} f_{m}(i x)\right|=\lim _{m, n \rightarrow \infty} \sup _{x \in \mathrm{R}}\left|f_{n}(i x)-f_{m}(i x)\right| .
$$

Since $f_{n}-f_{m}$ is bounded on $\overline{\mathrm{C}}^{+}$, a version of the maximum modulus principle in $\bar{C}^{+}$(for instance, the Phragmen-Lindelöff theorem for sectors) applies to obtain that $\left(f_{n}\right)$ is a Cauchy sequence uniformly on $\overline{\mathrm{C}}^{+}$. Hence $\left(f_{n}\right)$ converges 
uniformly on $\mathrm{C}^{+}$to a bounded and analytic function, say $g$. Obviously, $g=$ $e^{a z} G(z)$, so that $e^{a z} G(z)$ is bounded. Then by the density of $I$ we obtain that $e^{a z} \mathfrak{Y}^{(k)}\left(\mathrm{C}^{+}\right)$is formed exclusively by bounded functions, which is clearly false $\left(\right.$ take $\left.G(z)=(1+z)^{-1}\right)$.

Conversely, if for an ideal $I$ in $\mathfrak{A}^{(k)}\left(\mathrm{C}^{+}\right)$the set $\mathscr{Z}(I)$ is empty and the ideal is not dense then the inner parts of the elements of $I$ have a common inner divisor $Q$. The inner function $Q$ is nowhere vanishing, so it is of the form $e^{-a z}$ for some $a>0$, see [12]. Every element of $I$ is then of the form $e^{-a z} Q_{1} f$, where $Q_{1}$ is an inner function and $Q_{1} f$ is bounded. Hence $e^{a z} I$ consists of bounded functions. The proof follows.

\section{Sequences of higher order bounded variation and convolution}

In this section we introduce a discrete version of the algebras $\mathscr{T}_{+}^{n}\left(t^{n}\right)$ referred to in the Introduction. Let $(a(n))_{n=0}^{\infty}$ be a complex sequence. Throughout the section we shall consider the so-called difference operators $\Delta^{k}$ defined on $(a(n))_{n=0}^{\infty}$ by

$$
\left(\Delta^{0} a\right)(n):=a(n), \quad(\Delta a)(n):=a(n)-a(n+1)
$$

and

$$
\Delta^{1}=\Delta, \quad \Delta^{k+1}:=\Delta^{1} \circ \Delta^{k}=\Delta^{k} \circ \Delta^{1}
$$

for every non-negative integers $n$ and $k$. As a matter of fact $\Delta^{k}$ is explicitly given by

$$
\Delta^{k} a(n)=\sum_{j=0}^{k}(-1)^{j}\left(\begin{array}{l}
k \\
j
\end{array}\right) a(n+j) .
$$

The operator $\Delta$ is invertible on the space of sequences with finite support $c_{00}$, with inverse

$$
\Delta^{-1} a(n)=\sum_{j=n}^{\infty} a_{j}, \quad a \in c_{00} .
$$

An expression for the inverse $\Delta^{-k}$ of $\Delta^{k}$ will be given later.

We begin with a reproducing formula involving higher differences.

LeMma 4.1. Let $a(n)$ be a sequence of complex numbers with $\lim _{n} a(n)=0$. Then

$$
\sum_{n=1}^{\infty}\left|\Delta^{j} a(n)\right| n^{j} \leq \sum_{n=1}^{\infty}\left|\Delta^{j+1} a(n)\right| n^{j+1}, \quad \text { for every } \quad j \geq 0 .
$$


If, moreover,

$$
\sum_{n=1}^{\infty}\left|\Delta^{k} a(n)\right| n^{k}<\infty \quad \text { for some } k \geq 1
$$

then

(5) $\quad a(n)=\sum_{m=n}^{\infty} \frac{\Gamma(m-n+k)}{\Gamma(m-n+1) \Gamma(k)} \Delta^{k} a(m), \quad$ for every $\quad n \geq 0$.

Proof. To show (4), notice that for every $j, n \geq 0$ we have

$$
\Delta^{j} a(n)=\sum_{m=n}^{\infty}\left(\Delta^{j+1} a\right)(m)
$$

since $\Delta^{j+1} a(N)$ tends to 0 as $N \rightarrow \infty$. Hence,

$$
\begin{aligned}
\sum_{n=1}^{\infty}\left|\Delta^{j} a(n)\right| n^{j} & \leq \sum_{n=1}^{\infty} \sum_{m=n}^{\infty}\left|\Delta^{j+1} a(m)\right| n^{j}=\sum_{m=1}^{\infty}\left(\sum_{n=1}^{m} n^{j}\right)\left|\Delta^{j+1} a(m)\right| \\
& \leq \sum_{n=1}^{\infty}\left|\Delta^{j+1} a(m)\right| m^{j+1}
\end{aligned}
$$

since $\sum_{n=1}^{m} n^{j} \leq m^{j}\left(\sum_{n=1}^{m} 1\right)=m^{j+1}$.

Next, we prove (5). First notice that, by induction on $N$, it is readily seen that

$$
\sum_{j=0}^{N} \frac{\Gamma(j+k)}{\Gamma(j+1) \Gamma(k)}=\frac{\Gamma(N+k+1)}{\Gamma(N+1) \Gamma(k+1)} \quad(N \geq 0 ; k \geq 1) .
$$

Now we proceed by induction on $k$. Take $n$ a nonnegative integer. As before (for $j=0,1$ ),

$$
a(n)=\sum_{m=n}^{\infty}(\Delta a)(m)=\sum_{m=n}^{\infty} \sum_{p=m}^{\infty}\left(\Delta^{2} a\right)(p)
$$

so

$$
a(n)=\sum_{p=n}^{\infty}\left(\sum_{m=n}^{p} 1\right)\left(\Delta^{2} a\right)(p)=\sum_{p=n}^{\infty}(p-n+1)\left(\Delta^{2} a\right)(p) .
$$


Let assume that (5) holds for fixed $j$ with $0 \leq j<k$. Then

$$
\begin{aligned}
a(n) & =\sum_{m=n}^{\infty} \frac{\Gamma(m-n+j)}{\Gamma(m-n+1) \Gamma(j)} \Delta^{j} a(m) \\
& =\sum_{m=n}^{\infty} \frac{\Gamma(m-n+j)}{\Gamma(m-n+1) \Gamma(j)} \sum_{p=m}^{\infty} \Delta^{j+1} a(p) \\
& =\sum_{p=n}^{\infty}\left(\sum_{m=n}^{p} \frac{\Gamma(m-n+j)}{\Gamma(m-n+1) \Gamma(j)}\right) \Delta^{j+1} a(p) \\
& =\sum_{p=n}^{\infty} \frac{\Gamma(p-n+j+1)}{\Gamma(p-n+1) \Gamma(j+1)},
\end{aligned}
$$

where (6) has been used in the last equality (note that the exchange of summation order in the above calculation is justified by (4) and the finiteness assumption of the statement). The induction process has been completed.

The above result suggests introducing an operator, say $\Delta^{-k}$, in the following way.

For each sequence $(b(n))_{n=0}^{\infty}$ such that $\sum_{n=1}^{\infty}|b(n)| n^{k}<\infty$, put

$$
\left(\Delta^{-k} b\right)(n):=\sum_{m=n}^{\infty} \frac{\Gamma(m-n+k)}{\Gamma(m-n+1) \Gamma(k)} b(m), \quad(m \geq 0),
$$

Clearly, $\Delta^{-k} \circ \Delta^{k}=\mathrm{Id}=\Delta^{k} \circ \Delta^{-k}$ (for the second equality note that $\Delta \circ \Delta=\mathrm{Id}$ ), where we are assuming tacitly that both operators act on suitable sequences and Id is the corresponding identity operator.

Definition 4.2. Let $\tau^{k}\left(n^{k}\right)$ denote the vector space of complex sequences $a(n)$ such that $\lim _{n \rightarrow \infty} a(n)=0$ and $\sum_{n=1}^{\infty}\left|\Delta^{k} a(n)\right| n^{k}<\infty$.

Then $\tau^{k}\left(n^{k}\right)$ is a normed space with respect to the norm

$$
\|a\|_{\tau^{k}}:=|a(0)|+\sum_{n=1}^{\infty}\left|\Delta^{k} a(n)\right| n^{k} .
$$

Since $\Gamma(n+k+1) \Gamma(n+1)^{-1} \sim C_{k} n^{k}$, as $n \rightarrow \infty$ (for fixed $k$ ), the norm $\|a\|_{\tau^{k}}$ can be equivalently expressed by

$$
\|a\|_{\tau^{k}} \sim \sum_{n=0}^{\infty} \frac{\Gamma(n+k+1)}{\Gamma(n+1)}\left|\Delta^{k} a(n)\right| .
$$


We shall use this fact freely in the sequel.

By Lemma 4.1 and the remark prior to the above definition, the operators $\Delta^{k}: \tau^{k}\left(n^{k}\right) \rightarrow l^{1}\left((1+n)^{k}\right)$ and $\Delta^{-k}: l^{1}\left((1+n)^{k}\right) \rightarrow \tau^{k}\left(n^{k}\right)$ are inverse one of each other (continuous) mappings. In particular $\tau^{k}\left(n^{k}\right)$ is a Banach space.

From the estimate given in (4) one obtains the continuous inclusions

$$
\tau^{k+1}\left(n^{k+1}\right) \subseteq \tau^{k}\left(n^{k}\right) \subseteq \cdots \subseteq \tau^{1}(n) \subseteq \tau^{0}(1)=l^{1} \quad \text { for all } \quad k \geq 1 .
$$

Also, the space $c_{00}$ of sequences with finite support is dense in $\tau^{k}\left(n^{k}\right)$ since $\Delta^{k}\left(c_{00}\right)=c_{00}=\Delta^{-k}\left(c_{00}\right)$. So the sequences $\delta_{n}, n \in \mathrm{N}_{0}:=\mathrm{N} \cup\{0\}$, defined by $\delta_{n}(m)=1$, if $m=n, \delta_{n}(m)=0$, if $m \neq n$, generate the space $\tau^{k}\left(n^{k}\right)$. The norm of each $\delta_{n}$ in $\tau^{k}\left(n^{k}\right)$ is

$$
\left\|\delta_{n}\right\|_{\tau^{k}}=\sum_{m=n-k}^{n}\left(\begin{array}{c}
k \\
n-m
\end{array}\right) m^{k}=\sum_{j=0}^{k}\left(\begin{array}{l}
k \\
j
\end{array}\right)(n-j)^{k} \sim C_{k} n^{k},
$$

for $n \geq 1$, and $\left\|\delta_{0}\right\|_{\tau^{k}}=1$.

REMARK 4.3. The following sequences are relevant in our setting. For $m \geq$ 0 , put

$$
\Gamma_{m}^{k-1}:=\sum_{n=0}^{m} \frac{\Gamma(m-n+k)}{\Gamma(m-n+1) \Gamma(k)} \delta_{n} \in \tau^{k}\left(n^{k}\right) .
$$

The family $\left(\Gamma_{m}^{k-1}\right)_{m \geq 0}$ is to be considered in the present setting as a version, on $\mathrm{N}_{0}$, of the Riesz means on $(0, \infty)$, see [7] for instance. Let us find the norm of $\Gamma_{m}^{k-1}$ for each $m$.

Note that, for every $p \geq 0$,

$$
\Gamma_{m}^{k-1}(p)=\frac{\Gamma(m-p+k)}{\Gamma(m-p+1) \Gamma(k)} \quad \text { if } \quad 0 \leq p \leq m,
$$

whereas $\Gamma_{m}^{k-1}(p)=0$, if $p>m$. From this,

$$
\Gamma_{m}^{k-1}(p)=\sum_{n=p}^{\infty} \frac{\Gamma(n-p+k)}{\Gamma(n-p+1) \Gamma(k)} \delta_{m}(n) \quad(p \geq 0),
$$

and therefore $\Gamma_{m}^{k-1}=\Delta^{-k} \delta_{m}$ or, equivalently, $\Delta^{k} \Gamma_{m}^{k-1}=\delta_{m},(m \geq 0)$. Hence $\left\|\Gamma_{m}^{k-1}\right\|_{\tau^{k}}=\sum_{n=0}^{\infty}\left|\delta_{m}(n)\right| n^{k} \sim C_{k} m^{k}$.

The previous estimate is important because it enables us to express any $a \in \tau^{k}\left(n^{k}\right)$, in the norm of $\tau^{k}\left(n^{k}\right)$, as

$$
a=\sum_{m=0}^{\infty} \Delta^{k} a(m) \Gamma_{m}^{k-1}
$$


In fact, for a given $p \geq 0$, we have that $\Gamma_{m}^{k-1}(p)=0$ if $0<m \leq p-1$, and $\Gamma_{m}^{k-1}(p)=[\Gamma(n-p+1) \Gamma(k)]^{-1} \Gamma(n-p+k)$ when $m \geq p$. It follows that

$$
a(p)=\sum_{m=p}^{\infty} \frac{\Gamma(m-p+k)}{\Gamma(m-p+1) \Gamma(k)} \Delta^{k} a(m)=\sum_{m=p}^{\infty} \Delta^{k} a(m) \Gamma_{m}^{k-1}(p) .
$$

Finally, the last series converges in the norm of $\tau^{k}\left(n^{k}\right)$.

Next, in the second half of this section we prove that the Banach space $\tau^{k}\left(n^{k}\right)$ is a Banach algebra for the convolution. This result relies on the following formula.

Lemma 4.4. For every $a, b \in \tau^{k}\left(n^{k}\right), k \geq 0$ and $n=0,1, \ldots$,

$$
\begin{aligned}
\Gamma(k) \Delta^{k}(a * b)(n)= & \sum_{m=0}^{n} \Delta^{k} b(m) \sum_{p=n-m}^{n} \frac{\Gamma(p-n+m+k)}{\Gamma(p-n+m+1)} \Delta^{k} a(p) \\
& -\sum_{m=n+1}^{\infty} \Delta^{k} b(m) \sum_{p=n+1}^{\infty} \frac{\Gamma(p-n+m+k)}{\Gamma(p-n+m+1)} \Delta^{k} a(p) \\
= & : h(n) .
\end{aligned}
$$

Proof. First of all note that

$$
\begin{aligned}
h(n)= & \sum_{m=0}^{n} \Delta^{k} b(m) \sum_{p=n-m}^{\infty} \frac{\Gamma(p-n+m+k)}{\Gamma(p-n+m+1)} \Delta^{k} a(p) \\
& \quad-\sum_{m=0}^{\infty} \Delta^{k} b(m) \sum_{p=n+1}^{\infty} \frac{\Gamma(p-n+m+k)}{\Gamma(p-n+m+1)} \Delta^{k} a(p) \\
= & \sum_{m=0}^{n} \Delta^{k} b(m) \Gamma(k) a(n-m) \\
& \quad-\sum_{m=0}^{\infty} \Delta^{k} b(m) \sum_{p=n+1}^{\infty} \frac{\Gamma(p-n+m+k)}{\Gamma(p-n+m+1)} \Delta^{k} a(p),
\end{aligned}
$$

where in the last equality we have used (5). Thus

$$
\begin{aligned}
& h(n)=\Gamma(k)\left(\Delta^{k} b * a\right)(n) \\
& \quad-\sum_{m=0}^{\infty} \Delta^{k} b(m) \sum_{q=0}^{\infty} \frac{\Gamma(q+m+1+k)}{\Gamma(q+m+2)} \Delta^{k} a(q+n+1) .
\end{aligned}
$$


On the other hand,

$$
\begin{aligned}
\Delta^{-k}\left(\Delta^{k} b * a\right)(n) & \\
= & \frac{1}{\Gamma(k)} \sum_{m=n}^{\infty} \frac{\Gamma(m-n+k)}{\Gamma(m-n+1)}\left(\Delta^{k} b * a\right)(m) \\
= & \frac{1}{\Gamma(k)} \sum_{m=n}^{\infty} \frac{\Gamma(m-n+k)}{\Gamma(m-n+1)} \sum_{p=0}^{m} \Delta^{k} b(m-p) a(p) \\
= & \frac{1}{\Gamma(k)} \sum_{p=0}^{n} \sum_{m=n}^{\infty} \frac{\Gamma(m-n+k)}{\Gamma(m-n+1)} \Delta^{k} b(m-p) a(p) \\
& +\frac{1}{\Gamma(k)} \sum_{p=n+1}^{\infty} \sum_{m=p}^{\infty} \frac{\Gamma(m-n+k)}{\Gamma(m-n+1)} \Delta^{k} b(m-p) a(p) \\
= & (b * a)(n)+\frac{1}{\Gamma(k)} \sum_{p=n+1}^{\infty} \sum_{m=p}^{\infty} \frac{\Gamma(m-n+k)}{\Gamma(m-n+1)} \Delta^{k} b(m-p) a(p)
\end{aligned}
$$

Now, let pay attention to the second term of the last member in the above set of equalities. By expressing $a(p)$ using (5) and then rewriting indexes, we obtain

$$
\begin{aligned}
& \Delta^{-k}\left(\Delta^{k} b * a\right)(n)=(b * a)(n) \\
& \quad+\frac{1}{\Gamma(k)^{2}} \sum_{q, r, s=0}^{\infty} \Delta^{k} b(r) \frac{\Gamma(r+q+k+1)}{\Gamma(r+q+2)} \Delta^{k} a(s+q+n+1) \frac{\Gamma(s+k)}{\Gamma(s+1)}
\end{aligned}
$$

In summary, we have

$$
\begin{array}{rl}
\Delta^{-k} & h(n) \\
= & \Gamma(k)(b * a)(n) \\
& +\frac{1}{\Gamma(k)^{2}} \sum_{q, r, s=0}^{\infty} \Delta^{k} b(r) \frac{\Gamma(r+q+k+1)}{\Gamma(r+q+2)} \Delta^{k} a(s+q+n+1) \frac{\Gamma(s+k)}{\Gamma(s+1)} \\
& -\Delta^{-k}\left(\sum_{m, q=0}^{\infty} \Delta^{k} b(m) \frac{\Gamma(q+m+1+k)}{\Gamma(q+m+2)} \Delta^{k} a(q+(\cdot)+1)\right)(n)
\end{array}
$$


Moreover,

$$
\begin{gathered}
\Delta^{-k}\left(\sum_{m, q=0}^{\infty} \Delta^{k} b(m) \frac{\Gamma(q+m+1+k)}{\Gamma(q+m+2)} \Delta^{k} a(q+(\cdot)+1)\right)(n) \\
=\frac{1}{\Gamma(k)} \sum_{m, q=0}^{\infty} \frac{\Gamma(q+m+1+k)}{\Gamma(q+m+2)} \Delta^{k} b(m) \\
\left(\sum_{r=0}^{\infty} \frac{\Gamma(r+k)}{\Gamma(r+1)} \Delta^{k} a(r+q+n+1)\right) .
\end{gathered}
$$

In conclusion, $\Delta^{-k} h=\Gamma(k)(b * a)$; that is, $\Gamma(k) \Delta^{k}(b * a)=h$ as we wanted to show.

Note that, in the calculations of the above proof, we do not need to check the validity of exchanging the order of sums since it can be assumed that we work with sequences in the space $c_{00}$, which is dense in $\tau^{k}\left(n^{k}\right)$.

THEOREM 4.5. The space $\tau^{k}\left(n^{k}\right)$ is a Banach algebra with respect to the convolution product of sequences.

Proof. For $n \geq 0$ let $m, p$ such that either $0 \leq m \leq n$ and $n-m \leq p \leq n$, or $m, p>n$. Put $\gamma_{n, m, p}^{k}:=\Gamma(p-n+m+1)^{-1} \Gamma(p-n+m+k)$.

From (7) we obtain

$$
\begin{aligned}
\Gamma(k) & \sum_{n=0}^{\infty}\left|\Delta^{k}(a * b)(n)\right| n^{k} \\
& \leq\left(\sum_{n=0}^{\infty} \sum_{m=0}^{n} \sum_{p=n-m}^{n}+\sum_{n=0}^{\infty} \sum_{m=n+1}^{\infty} \sum_{p=n+1}^{\infty}\right) \gamma_{n, m, p}^{k}\left|\Delta^{k} b(m)\right|\left|\Delta^{k} a(p)\right| n^{k} \\
& =\left(\sum_{m, p=0}^{\infty} \sum_{n=\max \{p, m\}}^{m+p}+\sum_{m, p=1}^{\infty} \sum_{n=0}^{\min \{p, m\}-1}\right) \gamma_{n, m, p}^{k}\left|\Delta^{k} b(m)\right|\left|\Delta^{k} a(p)\right| n^{k},
\end{aligned}
$$

where, to obtain the equality, we have applied Fubini's rule twice.

Since, moreover,

$$
\sum_{n=\max \{p, m\}}^{m+p} \gamma_{n, m, p}^{k} n^{k} \leq(p+m)^{k} \sum_{n=m}^{m+p} \gamma_{n, m, p}^{k} \sim C_{k} m^{k} p^{k}
$$

and

$$
\sum_{n=0}^{\min \{p, m\}-1} \gamma_{n, m, p}^{k} n^{k} \leq \min \{p, m\}^{k} \sum_{n=0}^{m+p} \gamma_{n, m, p}^{k} \sim C_{k} m^{k} p^{k}
$$


one gets

$$
\sum_{n=0}^{\infty}\left|\Delta^{k}(a * b)(n)\right| n^{k} \leq C\left(\sum_{n=0}^{\infty}\left|\Delta^{k} a(n)\right| n^{k}\right)\left(\sum_{n=0}^{\infty}\left|\Delta^{k} b(n)\right| n^{k}\right)
$$

for all $a, b \in \tau^{k}\left(n^{k}\right)$, which means that $\tau^{k}\left(n^{k}\right)$ is a Banach algebra.

\section{The image of $\tau^{k}\left(n^{k}\right)$ in the Wiener algebra}

Since $\tau^{k}\left(n^{k}\right)$ is densely contained in $l^{1}$ and this inclusion is also continuous, each character of $l^{1}$ gives rise to a character of $\tau^{k}\left(n^{k}\right)$ of the form

$$
\chi_{z}: a \mapsto \chi_{z}(a):=\hat{a}(z)=\sum_{n=0}^{\infty} a(n) z^{n}, \quad \tau^{k}\left(n^{k}\right) \rightarrow \mathrm{C}
$$

where $|z| \leq 1$. We are going to see that every character of $\tau^{k}\left(n^{k}\right)$ is like the above one. Recall that the sequence $\Gamma_{m}^{k-1}$ in $\tau^{k}\left(n^{k}\right)$ is defined as

$$
\Gamma_{m}^{k-1}:=\sum_{n=0}^{m} \frac{\Gamma(m-n+k)}{\Gamma(m-n+1) \Gamma(k)} \delta_{n} .
$$

where the convergence is in the norm of $\tau^{k}\left(n^{k}\right)$.

Proposition 5.1. The set of characters, or equivalently the spectrum of maximal ideals, of $\tau^{k}\left(n^{k}\right)$ is identified with $\overline{\mathrm{D}}$, and its Gelfand transform $\mathscr{G}$ is given by

$$
\mathscr{G}: a \mapsto \mathscr{G}(a):=\hat{a}, \quad \tau^{k}\left(n^{k}\right) \rightarrow A^{+}(\mathrm{T}),
$$

where

$$
\hat{a}(z)=\sum_{m=0}^{\infty} \Delta^{k} a(m) \widehat{\Gamma_{m}^{k-1}}(z)=\sum_{n=0}^{\infty} a(n) z^{n}, \quad|z| \leq 1 .
$$

PROOF. We only need to show that any character is of the form $\chi_{z}$, for some $z$ with $|z| \leq 1$. This is standard but not obvious. So, let $\chi$ be a character of $\tau^{k}\left(n^{k}\right)$ and take $z:=\chi\left(\delta_{1}\right)$. From the multiplicativity property, we have that $\left|\chi\left(\delta_{n}\right)\right|=\left|\chi\left(\delta_{1}\right)^{n}\right|=|z|^{n}$ for every $n \in \mathrm{N}_{0}$. On the other hand, $\left|\chi\left(\delta_{n}\right)\right| \leq$ $C\left\|\delta_{n}\right\|_{\tau^{k}} \sim n^{k}$. It follows that $|z|^{n} \leq C n^{k}$ for all $n \geq 1$, whence $|z| \leq 1$. Hence, for all $m \geq 0$,

$$
\chi\left(\Gamma_{m}^{k-1}\right)=\sum_{n=0}^{m} \frac{\Gamma(m-n+k)}{\Gamma(m-n+1) \Gamma(k)} z^{n} .
$$


Recall now that $a=\sum_{m=0}^{\infty} \Delta^{k} a(m) \Gamma_{m}^{k-1}$ in the norm of $\tau^{k}\left(n^{k}\right)$. Thus

$$
\begin{aligned}
\chi(a) & =\sum_{m=0}^{\infty} \Delta^{k} a(m) \sum_{n=0}^{m} \frac{\Gamma(m-n+k)}{\Gamma(m-n+1) \Gamma(k)} z^{n} \\
& =\sum_{n=0}^{\infty} \sum_{m=n}^{\infty} \frac{\Gamma(m-n+k)}{\Gamma(m-n+1) \Gamma(k)} \Delta^{k} a(m) z^{n}=\sum_{n=0}^{\infty} a(n) z^{n}=: \chi_{z}(a),
\end{aligned}
$$

as we wanted to show.

Now we characterize the range of the Gelfand transform $\mathscr{G}: \tau^{k}\left(n^{k}\right) \rightarrow$ $A_{+}(\mathrm{T})$.

Lemma 5.2. Let $x_{0}, x_{1}, \ldots, x_{k}, y_{0}, y_{1}, \ldots, y_{k} \in \mathrm{C}$. Then

$$
y_{p}=\sum_{m=0}^{p}\left(\begin{array}{c}
p \\
m
\end{array}\right) x_{m} \quad(p=0,1, \ldots, k)
$$

if and only if

$$
x_{m}=\sum_{p=0}^{m}(-1)^{m+p}\left(\begin{array}{c}
m \\
p
\end{array}\right) y_{p} \quad(m=0,1, \ldots, k)
$$

Proof. Let $\left(\alpha_{p q}\right),\left(\beta_{p q}\right)$ be the lower triangular matrices defined by

$$
\beta_{p q}=(-1)^{p+q} \alpha_{p q} \quad \text { and } \quad \alpha_{p q}= \begin{cases}\left(\begin{array}{l}
p \\
q
\end{array}\right), & \text { if } p \geq q \\
0, & \text { if } p<q .\end{cases}
$$

Then the product matrix $\left(\gamma_{p q}\right):=\left(\alpha_{p q}\right) \cdot\left(\beta_{p q}\right)$ turns out to be the identity matrix: For $p<q, \gamma_{p q}=0$ clearly; for $p \geq q \geq 0$,

$$
\begin{aligned}
\gamma_{p q} & =\sum_{j=0}^{p-q}\left(\begin{array}{c}
p \\
q+j
\end{array}\right)(-1)^{j}\left(\begin{array}{c}
q+j \\
q
\end{array}\right)=\sum_{j=0}^{p-q}(-1)^{j} \frac{p !}{j ! q !(p-q-j) !} \\
& =\left(\begin{array}{c}
p \\
q
\end{array}\right) \sum_{j=0}^{p-q}(-1)^{j}\left(\begin{array}{c}
p-q \\
j
\end{array}\right)=\left(\begin{array}{c}
p \\
q
\end{array}\right)(1-1)^{p-q} \\
& = \begin{cases}1, & \text { if } p=q ; \\
0, & \text { if } p>q .\end{cases}
\end{aligned}
$$

Analogously, $\left(\beta_{p q}\right) \cdot\left(\alpha_{p q}\right)=(-1)^{p+q}\left(\alpha_{p q}\right),\left(\beta_{p q}\right)$ is also the identity matrix. This of course implies the statement. 
For a complex sequence $a$ and $n, p \geq 0$, put

$$
b_{p}(n):=\left(\begin{array}{c}
n+p \\
n
\end{array}\right) \Delta^{p} a(n) .
$$

For a natural number $k$ and $a \in \tau^{k}\left(n^{k}\right)$ we know that $\sum_{n=0}^{\infty}\left|b_{p}(n)\right|<\infty$ provided $p=0,1, \ldots, k$.

Proposition 5.3. Let $k$, $a$ and $b_{p}$ be as above. Then

$$
\hat{b}_{p}(z)=\frac{1}{m !} \sum_{m=0}^{p}\left(\begin{array}{c}
p \\
m
\end{array}\right)(z-1)^{m} \hat{a}^{(m)}(z), \quad \text { if } \quad|z| \leq 1,
$$

$(p=0,1, \ldots, k)$ and

$$
\frac{1}{m !}(z-1)^{m} \hat{a}^{(m)}(z)=\sum_{p=0}^{m}(-1)^{p+m}\left(\begin{array}{c}
m \\
p
\end{array}\right) \hat{b}_{p}(z), \quad \text { if } \quad|z| \leq 1
$$

$(m=0,1, \ldots, k)$.

Proof. We begin with proving (8). For $a$ in $\tau^{k}\left(n^{k}\right), z \in \mathrm{C}$ such that $|z| \leq 1$ and $0 \leq p \leq k$,

$$
\begin{aligned}
\hat{b}_{p}(z) & :=\sum_{n=0}^{\infty}\left(\begin{array}{c}
n+p \\
n
\end{array}\right) \Delta^{p} a(n) z^{n} \\
& =\sum_{j=0}^{p}(-1)^{j}\left(\begin{array}{l}
p \\
j
\end{array}\right) \sum_{n=0}^{\infty}\left(\begin{array}{c}
n+p \\
n
\end{array}\right) a(n+j) z^{n} \\
& =\frac{1}{p !} \sum_{j=0}^{p}(-1)^{j}\left(\begin{array}{l}
p \\
j
\end{array}\right)\left(\sum_{n=0}^{\infty} a(n+j) z^{n+p}\right)^{(p)} \\
& =\frac{1}{p !} \sum_{j=0}^{p}(-1)^{j}\left(\begin{array}{l}
p \\
j
\end{array}\right)\left[z^{p-j} \sum_{n=0}^{\infty} a(n+j) z^{n+j}\right]^{(p)} \\
& =\frac{1}{p !} \sum_{j=0}^{p}(-1)^{j}\left(\begin{array}{l}
p \\
j
\end{array}\right) \sum_{m=0}^{p}\left(\begin{array}{c}
p \\
m
\end{array}\right)\left(z^{p-j}\right)^{(p-m)}\left(\sum_{n=0}^{\infty} a(n+j) z^{n+j}\right)^{(m)} \\
& =\frac{1}{p !} \sum_{j=0}^{p}(-1)^{j}\left(\begin{array}{l}
p \\
j
\end{array}\right) \sum_{m=j}^{p}\left(\begin{array}{c}
p \\
m
\end{array}\right) \frac{(p-j) !}{(m-j) !} z^{m-j} \hat{a}^{(m)}(z)
\end{aligned}
$$




$$
\begin{aligned}
& =\sum_{m=0}^{p} \frac{1}{m !}\left(\begin{array}{c}
p \\
m
\end{array}\right) \sum_{j=0}^{m}(-1)^{j}\left(\begin{array}{c}
m \\
j
\end{array}\right) z^{m-j} \hat{a}^{(m)}(z) \\
& =\frac{1}{m !} \sum_{m=0}^{p}\left(\begin{array}{c}
p \\
m
\end{array}\right)(z-1)^{m} \hat{a}^{(m)}(z) .
\end{aligned}
$$

So we have proved (8). Moreover, (8) is exactly the first equality of Lemma 5.2 for $y_{p}:=\hat{b}_{p}(z)$ and $x_{m}:=(1 / m !)(z-1)^{m} \hat{a}^{(m)}(z)$. Hence (9) follows now by Lemma 5.2.

The above proposition allows us to obtain the image of the Gelfand transform of $\tau^{k}\left(n^{k}\right)$ almost immediately. Let $\mathscr{H}_{1}^{(k),+}(\mathrm{T})$ denote the (pointwise multiplication) subalgebra of $A(D)$ formed by those functions $f$ such that

$$
(1-z)^{m} f^{(m)}(z) \in A^{+}(\mathbf{T}) \quad(m=0,1, \ldots, k),
$$

and provided with the algebra norm

$$
\|f\|:=\sum_{m=0}^{k}\left\|(z-1)^{m} f^{(m)}\right\|_{A^{+}(\mathrm{T})} .
$$

Note that the above condition implies that the value of each $(1-z)^{m} f^{(m)}$ at $z=1$, for $m=1, \ldots, k$, is indeed 0 , according to Corollary 2.3. In particular we have that $\mathscr{H}_{1}^{(k),+}(\mathrm{T})$ is a subalgebra of the algebra $\mathscr{H}_{1}^{(k)}(\mathrm{D})$ introduced in Section 2.

THEOREM 5.4. The Gelfand transform is a topological isomorphism betwen the Banach algebras $\tau^{k}\left(n^{k}\right)$ and $\mathscr{H}_{1}^{(k),+}(\mathrm{T})$.

Proof. If $a \in \tau^{k}\left(n^{k}\right)$ then (9) implies that $\hat{a} \in \mathscr{H}_{1}^{(k),+}(\mathrm{T})$ and that the norm of $\hat{a}$ is dominated by a constant times the norm of $a$ in $\tau^{k}\left(n^{k}\right)$. Conversely, given an element $f$ in $\mathscr{H}_{1}^{(k),+}(\mathrm{T})$, if we take $a(n):=\hat{f}(n)$ for all $n \geq 0$ (where $\hat{f}$ is the $n$-th Fourier coefficient of $f$ ), then (8) entails that $a \in \tau^{k}\left(n^{k}\right)$ and that the norm of $a$ is dominated by the norm of $f$. This implies the required isomorphism (and that $\mathscr{H}_{1}^{(k),+}(\mathrm{T})$ is complete, in particular).

REMARK 5.5. The preceding results provide us with another way to show that $\tau^{k}\left(n^{k}\right)$ is a convolution Banach algebra. In fact, the multiplicative structures are not needed here in order to prove the underlined (topological) linear isomorphism given in Theorem 5.4, for we can think of $\mathscr{G}$ just as the Taylor series mapping associated with the sequences of $\tau^{k}\left(n^{k}\right)$. Since conditions (10) and (11) yield by themselves, and automatically, a Banach algebra (that one 
denoted here by $\mathscr{H}_{1}^{(k),+}(\mathrm{T})$ ), and pointwise multiplication in $\mathscr{H}_{1}^{(k),+}(\mathrm{T})$ corresponds to convolution in $\tau^{k}\left(n^{k}\right)$, the linear version of Theorem 5.4 is enough to imply that $\tau^{k}\left(n^{k}\right)$ is a Banach algebra. However, formula (7) or the introduction of elements like $\Gamma_{m}^{k-1}$ seem to be of interest in their own.

\section{Standard ideals in Wiener-like algebras}

In this section we show that closed ideals in $\tau^{k}\left(n^{k}\right)$ with countable hull $Z(I)$ are standard, by following a discussion similar to that one of Sections 2 and 3 . The role that the Korenblyum algebra played in Section 2 is to be played here by the weighted Wiener algebra $A_{k}^{+}(\mathrm{T})$ defined in Section 1 . Recall that $F \in A_{k}^{+}(\mathrm{T})$ means that $\sum_{n=0}^{\infty}|\hat{F}(n)|\left(1+n^{k}\right)<\infty$, and that it is equivalent to have $F^{(j)} \in A^{+}(\mathrm{T})$ for every $j=0, \ldots, k$.

Put $\mathscr{I}_{1}^{(k-1),+}(\mathrm{T}):=\left\{F \in A_{k}^{+}(\mathrm{T}): F^{(j)}(1)=0,0 \leq j \leq k-1\right\}$. Clearly, $\mathscr{I}_{1}^{(k-1),+}(\mathrm{T})$ is contained in the ideal $\mathscr{I}_{1}^{(k-1)}(\mathrm{D})$ defined in Section 2.

THEOREM 6.1. For every non negative integer $k$, we have

$$
(1-z)^{k} \mathscr{H}_{1}^{(k),+}(\mathrm{T})=\mathscr{I}_{1}^{(k-1),+}(\mathrm{T})
$$

Proof. The fact that $(1-z)^{k} f \in \mathscr{I}_{1}^{(k-1),+}(\mathrm{T})$ if $f \in \mathscr{H}_{1}^{(k),+}(\mathrm{T})$ is an obvious consequence of the Leibniz' rule for derivatives.

Conversely, let $F$ be a function of $\mathscr{I}_{1}^{(k-1),+}(\mathrm{T})$. Take $f(z)=F(z)(1-z)^{-k}$ for $|z|<1$. Then $f$ is holomorphic in $\mathrm{D}$. We are going to show that $f$ lies in $A^{+}(\mathrm{T})$. Fix $r$ with $0<r<1$. Then, for each $n$,

$$
\begin{aligned}
\hat{f}(n) & =\frac{1}{2 \pi i} \int_{|z|=r} \frac{f(z)}{z^{n+1}} d z=\frac{1}{2 \pi i} \int_{|z|=r} \frac{F(z)}{(1-z)^{k} z^{n+1}} d z \\
& =\frac{1}{2 \pi i} \int_{|z|=r} F(z) \sum_{m=0}^{\infty} \frac{\Gamma(m+k)}{\Gamma(m+1)} z^{m-n-1} d z \\
& =\frac{1}{2 \pi i} \int_{|z|=r} F(z) \sum_{m=0}^{n} \frac{\Gamma(m+k)}{\Gamma(m+1)} z^{m-n-1} d z \\
& =\sum_{m=0}^{n} \frac{\Gamma(m+k)}{\Gamma(m+1)} \hat{F}(n-m)=\sum_{m=0}^{n} \frac{\Gamma(n-m+k)}{\Gamma(n-m+1)} \hat{F}(m) .
\end{aligned}
$$

On the other hand, we have

$$
F^{(j)}(z)=\sum_{m=j}^{\infty} \hat{F}(m) j !\left(\begin{array}{c}
m \\
j
\end{array}\right) z^{m}, \quad|z| \leq 1,
$$


for every $j=0,1, \ldots, k-1$. Hence $0=F^{(j)}(1)=j ! \sum_{m=j}^{\infty} \hat{F}(m)\left(\begin{array}{c}m \\ j\end{array}\right)$. From this we readily obtain that

$$
\sum_{m=0}^{\infty} \hat{F}(m) P(m)=0
$$

for every polynomial function $P$ of degree strictly less than $k$. For every $n$, take the polynomial $P_{n}(x)=(n-x+k-1) \ldots(n-x+1)$. Then

$$
\begin{aligned}
\sum_{n=0}^{\infty}|\hat{f}(n)| & =\sum_{n=0}^{\infty}\left|\sum_{m=0}^{n} \frac{\Gamma(n-m+k)}{\Gamma(n-m+1)} \hat{F}(m)\right| \\
& =\sum_{n=0}^{\infty}\left|\sum_{m=0}^{n} P_{n}(m) \hat{F}(m)\right|=\sum_{n=0}^{\infty}\left|-\sum_{m=n+1}^{\infty} P_{n}(m) \hat{F}(m)\right| \\
& =\sum_{n=0}^{\infty}\left|(-1)^{k-1} \sum_{m=n+1}^{\infty} \frac{\Gamma(m-n)}{\Gamma(m-n-k+1)} \hat{F}(m)\right| \\
& \leq \sum_{m=0}^{\infty}\left(\sum_{n=0}^{m-1} \frac{\Gamma(m-n)}{\Gamma(m-n-k+1)}\right)|\hat{F}(m)|<\infty
\end{aligned}
$$

since $\sum_{n=0}^{m-1} \frac{\Gamma(m-n)}{\Gamma(m-n-k+1)} \simeq C_{k}(m+1)^{k}$.

In short, we have shown, for all nonnegative integer $k$, that

$$
\text { if } F \in \mathscr{I}_{1}^{k-1,+}(\mathrm{T}) \text { and } f=(1-z)^{-k} F \text { then } f \in A^{+}(\mathrm{T}) \text {. }
$$

To conclude, we must prove that $(1-z)^{m} f^{(m)} \in A^{+}(\mathrm{T})$ for $1 \leq m \leq k$. So, fix $m$ such that $1 \leq m \leq k$ and set $f_{m}:=(1-z)^{m} f^{(m)}$. Then, for $z \in \mathbf{T} \backslash\{1\}$,

$$
f_{m}(z)=\sum_{j=0}^{m}\left(\begin{array}{c}
m \\
j
\end{array}\right) \frac{(k+j-1) !}{(k-1) !}(1-z)^{-(k-m+j)} F^{(m-j)}
$$

where $F^{(m-j)} \in \mathscr{I}_{1}^{(k-m+j-1,+)}$ for $j=0, \ldots, m$. From (12) we have that each $(1-z)^{-(k-m+j)} F^{(m-j)}$ in the finite sum belongs to $A^{+}(\mathrm{T})$. This means that $f_{m} \in A^{+}(\mathrm{T})$, and so the proof is over.

The theorem enables us to perform the kind of argument given in Section 2 to conclude that the closed ideals of $\mathscr{H}_{1}^{(k),+}(\mathrm{T})$ can be described in terms of the closed ideals of $A_{k}^{+}(\mathrm{T})$. In general the closed ideals of $A_{k}^{+}(\mathrm{T})$ are far from being completely understood, but we can determine them in some cases, see [2]. In 
the following result $J\left(Q ; \mathfrak{S}_{0}, \ldots, \mathfrak{S}_{k}\right)$ has the same meaning as in Theorem 2.5 , only that now the elements of $J\left(Q ; \mathfrak{S}_{0}, \ldots, \mathfrak{S}_{k}\right)$ belong to $\mathscr{H}_{1}^{(k),+}(\mathrm{T})$.

THEOREM 6.2. Every closed ideal I of $\mathscr{H}_{1}^{(k),+}(\mathrm{T})$ has the form

$$
I=(z-1)^{-k} J
$$

for some closed ideal of $A_{k}^{+}(\mathrm{T})$ contained in $\mathscr{I}_{1}^{(n-1),+}(\mathrm{T})$. Hence, if I has (at most) countable hull then it is standard, that is, of the form

$$
I=J\left(Q ; \mathfrak{S}_{0}, \ldots, \mathfrak{S}_{k}\right) .
$$

Proof. The mapping $f \mapsto(1-z)^{k} f, \mathscr{H}_{1}^{(k),+}(\mathrm{T}) \rightarrow \mathscr{I}_{1}^{(k-1),+}(\mathrm{T})$ is clearly continuous and injective, and is also surjective by Theorem 6.1. Thus it is a Banach $A_{k}^{+}(\mathrm{T})$-module isomorphism, so that $J:=(z-1)^{k} I$ is a closed ideal of $A_{k}^{+}(\mathrm{T})$ contained in $\mathscr{I}_{1}^{(k-1),+}(\mathrm{T})$. It follows that $I=(z-1)^{-k} J$.

Now assume that the hull $Z(I)$ is at most countable. Then $Z(J)$ is at most countable as well, and therefore it is standard in accordance with Theorem 3.1 of [2]. It follows that $I$ is standard as in the statement.

\section{REFERENCES}

1. Arendt, W., and Kellerman, H., Integration solutions of Volterra integrodifferential equations and applications, pp. 21-51 in: G. Da Prato, M. Iannelli (Eds.), Volterra Integrodifferential Equations in Banach Spaces and Applications, Proc. Trento 1987, Pitman Res. Notes Math. 190, Longman, Harlow 1989.

2. Agrafeuil, C., and Zarrabi, M., Closed ideals with countable hull in algebras of analytic functions smooth up to the boundary, Publ. Mat. 52 (2008), 19-56.

3. El Fallah, O., Ideaux fermés de $L^{1}\left(\mathrm{R}_{+}\right)$, Math. Scand. 72 (1993), 120-130.

4. Esterle, J., Distributions on Kronecker sets, strong forms of uniqueness, and closed ideals of $A^{+}$, J. reine angew. Math. 450 (1994), 43-82.

5. Esterle, J., Strouse, E., and Zouakia, F., Closed ideals of $A^{+}$and the Cantor set, J. reine angew. Math. 449 (1994), 65-79.

6. Galé, J. E., and Miana, P. J., One-parameter groups of regular quasimultipliers, J. Funct. Anal. 237 (2006), 1-53.

7. Galé, J. E., Miana, P. J., and Royo, J. J., Nyman type theorem in convolution Sobolev algebras, Rev. Mat. Complut. (to appear).

8. Galé, J. E., Miana, P. J., and Royo, J. J., Estimates of the Laplace transform on fractional Banach algebras, submitted.

9. Gasper, G., and Trebels, W., Multiplier criteria of Hörmander type for Fourier series and applications to Jacobi series and Hankel transforms, Math. Ann. 242 (1979), 225-240.

10. Gurariŭ, V. P., Spectral synthesis of bounded functions on the half axis (Russian), Funkcional. Anal. i Priložen 3:4 (1969), 34-48.

11. Hendenmalm, H., A comparison between the closed modular ideals in $l^{1}(w)$ and $L^{1}(w)$, Math. Scand. 58 (1986), 275-300.

12. Hoffman, K., Banach Spaces of Analytic Functions, Prentice-Hall, Englewood Cliffs, NJ 1962. 
13. Korenbljum, B. I., Closed ideals of the ring $A^{n}$ (Russian), Funkcional. Anal. i Priložen. 6:3 (1972), 38-52.

14. Miana, P. J., $\alpha$-times integrated semigroups and fractional derivation, Forum Math. 14 (2002), 23-46.

15. Pedersen, T. V., Closed ideals and the Bennet-Gilbert conjecture in Banach algebras of analytic functions, Arch. Math. (Basel) 70 (1998), 391-398.

\author{
DEPARTAMENTO DE MATEMÁTICAS E IUMA \\ UNIVERSIDAD DE ZARAGOZA \\ 50009 ZARAGOZA \\ SPAIN \\ E-mail: gale@unizar.es
}

\author{
DEPARTAMENTO DE MATEMÁTICAS \\ UNIVERSIDAD AUTÓNOMA \\ METROPOLITANA-IZTAPALAPA AP 55-534 \\ 09340 MÉXICO D. F. \\ MÉXICO \\ E-mail: awaw@xanum.uam.mx
}

